\title{
STUDY OF SOME HEALTH EFFECTS IN WORKERS EXPOSED TO CHLOROFORM
}

\author{
By \\ Zawilla, N.H.* and Hassanin, L.A.**
}

FROM

* Industrial Medicine and Occupational Diseases Dep., Faculty of Medicine, Cairo University. ** National Organization for Drug Control and Research (NODCAR).

\begin{abstract}
Chloroform (also known as trichloromethane or methyltrichloride), is a colorless liquid with a pleasant, non-irritating odor.

Most of chloroform found in the environment comes from industry; it is also found in waste water from sewage treatment plants and drinking water to which chlorine is added. Chloroform is used in pesticide formulation, in drugs and chemical industry and as a laboratory reagent. Chloroform toxicity can be attributed to the presence of both the parent compound and the formation of reactive metabolite (phosgene). Chloroform has obvious hepatic - nephrotoxicity and is a suspected human carcinogen. The genotoxic potential of chloroform has been investigated in a number of studies both in vitro and in vivo.

Also, the mechanism of action of chloroform had been investigated, several modes of actions were studied, of which cytotoxicity and cell replication, induction of gene mutation and DNA damage, lipid peroxidation and antioxidants depletion are the most common effects.

Objectives: (1) To study and evaluate some of the health effects of chronic occupational exposure to chloroform when used as a main laboratory reagent, with special emphasis on hepatorenal, and cytogenetic effects. (2) Also, assessment of possible effect of chloroform in causing oxidative stress and lipid peroxidation.
\end{abstract}


Methods: This study was carried out in the laboratory of Analgesic and Narcotic Drugs Department in the National Organization for Drug Control and Research. The study population was composed of 52 subjects; 30 workers were exposed to chloroform (by inhalation and skin contact) and 22 matched unexposed females were included as control.

Every individual in the study was subjected to clinical examination and special questionnaire, investigation of liver and kidney functions (ALT, AST, ALK phosphatase, bilirubin, serum creatinine and blood urea). Serum malondialdehyde (MDA) was measured for assessment of lipid peroxidation, and chromosomal simple analysis study was done.

Results: As regards the significant clinical manifestations, sensory disturbances, skin manifestations (redness, itching and allergic dermatitis), and cardio-vascular manifestations (arrhythmia and syncope) were elicited in 50\% of the exposed workers, and musculoskeletal manifestation were also found in the exposed group with statistically significant difference when compared to control.

Liver and kidney function parameters did not show statistically significant difference between the two groups, while serum MDA was statistically significantly higher in the exposed group $(\mathrm{P}<0.05)$. Chromosomal aberrations were significantly higher in the exposed subjects. However, correlations duration of work with MDA level, and with chromosomal aberrations, and MDA level with chromosomal aberrations were non-significant,

Conclusion: Occupational exposure to chloroform as a main laboratory reagent, was associated with significant increase in lipid peroxidation indicated by increased level of a lipid peroxidation product, serum MDA, and was associated with increased chromosomal aberrations that did not correlate with the level of serum MDA or with duration of exposure. Hepatorenal function parameters were within normal, skin manifestation arrhythmia, and sensory manifestations are the most significant clinical effects.

\section{Introduction}

Chloroform is a colorless liquid with a pleasant, non-irritating odor and a slightly sweet taste.

Chloroform is used in pesticide formulations, in drugs and flavors, and it is used as a general laboratory reagent. Also, it is used as an intermediate in chemical industry.

Chloroform is manufactured by hydrochlorination of methanol or by chlorination of methane (Xu and Weisel, 2005).

Chloroform is well absorbed in animals and humans after oral administra- 
tions, $60-80 \%$ of chloroform is absorbed after inhalation, dermal absorption is also a significant route. Distribution of chloroform occurs throughout the whole body. Highest tissue levels are reached in the fat, blood, liver, kidneys, lungs, and nervous system (Erdinger et al. 2004).

Oxidative biotransformation of chloroform is catalyzed by the cytochrome P450 to produce trichloromethanol. Phosgene is produced as a reactive intermediate. The interaction of phosgene with tissues proteins causes cellular degeneration and death (Gemma et al., 2003).

The liver and kidney are the target organs for acute toxicity of chloroform. Fatty infiltrations, cell ballooning, necrosis, may occur (Yaun et al., 2005).

The carcinogenic effect of chloroform appears to be closely related to cytotoxicity and regeneration cellular replication observed in the target organs (Lévesque et al. 2002). The ACGIH considered chloroform as a suspected human carcinogen (A2 substance) (ACGIH, 1994 and Rosenberg \& Katz, 2004).

The chronic toxicity of chloroform is well established because of its long history of use as an anesthetic. Inhalation of $10.000 \mathrm{ppm}$ of chloroform vapor produces clinical anesthesia, death may occur from cardio vascular depression and ventricular fibrillation, or from respiratory failure, delayed death may occur from acute hepatic necrosis (Gemma et al., 2003).

The genotoxic potential of chloroform has been investigated in a number of studies both in vitro and in vivo. Although there were clearly positive results in a few genotoxicity assays, chloroform was more frequently found to be negative than positive in genotoxicity tests, and is considered as an epigenetic carcinogen by some scientists (Meek et al., 2002).

According to Harris and Groh (1985), chloroform exerts its neurotoxic and respiratory effects through interaction with gangliosides in neuronal membranes and phospholipids in the surfactant monolayer of the lower respiratory tract.

Beddowes et al. (2003) deduced that chloroform may induce an oxidative stress via depletion of glutathione GSH and other antioxidant defenses; lipid peroxidation follows with increase in lipid peroxidation products e.g. MDA (malondialdlehyde).

\section{Aim of this study}

This study was conducted to evaluate some of the health effects of chronic occupational exposure to chloroform, with special emphasis on hepatorenal, and cytogenetic effects. In addition this work was 
aimed to assess the possible effect of exposure to chloroform in causing oxidative stress and lipid peroxidation.

\section{Subjects and methods}

\section{Subjects:}

The study population was composed of 52 individuals divided into exposed and control groups.

The exposed groups were composed of 30 females working as biochemists, pharmacists and technicians in the laboratory of Analgesic and Narotic Drugs Department in National Organization for Drug Control and Research [NODCR].

They were exposed to chloroform which is used in operation of the HPLC and in extraction for analysis of drugs.

Protective devices were not used, except for occasional use of gloves.

The control group was composed of 22 females, working as clerks and office personnel, selected by random sampling and not exposed to chloroform or any solvents, or genotoxic agents in their workplace.

They were matched as regards age, sex, and socioeconomic status to the exposed group.

All the studied subjects were nonsmokers.

\section{Methods:}

Every individual in this study was subjected to the following:-

\section{1- Questionnaire:}

A specially designed questionnaire including personal, occupational, medical and genetic history.

\section{2- Thorough clinical examination:}

\section{3- Laboratory investigations:}

Fifteen $\mathrm{ml}$ of blood were withdrawn from each individual. Five $\mathrm{ml}$ of them were taken on heparin for cytogentic analysis, and the other $10 \mathrm{ml}$ were allowed to clot then centrifuged for assessment of liver and kidney functions and serum malondialdehyde.

A- Liver and kidney functions:

Total bilirubin, alanine transaminase (ALT), aspartate transaminase (AST), alkaline phosphatase, blood urea, and serum creatinine, were estimated using Hilashi (119) auto analyzer. La Roche, Germany, supplied the kits and instrument.

B- Malondialdehyde (MDA):

Serum MDA was assayed using the standard technique described by (Stringer et al., 1989).

\section{4- Cytogenetic analysis:-}

The collected blood samples were kept 
in an upright position for two hours then cultured on a culture medium (RPM1 1640 gibco) + phytohaemoglutinin, supplemented with L-glutamine and fetal calf serum. Cultures were incubated at $37^{\circ} \mathrm{C}$ for 72 hours. Colchicine was added 30 minutes prior to harvest to a final concentration of $0.1 \mathrm{mg} \backslash \mathrm{ml}$. The cultures were centrifuged for 10 minutes and the supernatant fluid was discarded. Hypotonic solution was added to cultures and kept for 30 minutes at $37^{\circ} \mathrm{C}$. Then the cultures were centrifuged for 10 minutes and the supernatant fluid was discarded. The cultured cells were then washed by addition of a fixative solution (methanol: glacial acetic). The process of fixing cells was repeated for 3-4 times. After the last time of washing, part of the supernatant fluid was discarded and the rest was mixed with the ppt. Four to six drops are dropped on a cold wet slide and left to dry. The slides were stained for 5 minutes in 10\% Gimsa stain in $\mathrm{pH} 6.8$ (Verma and Babu, 1989).

\section{Statistical Analysis}

Results were evaluated for each group. Data were compared using different tests according to the type of data. The unpaired student " $\mathrm{t}$ " test was used for comparing the means of both groups. The chi-square test was used to perform qualitative comparison between the different groups. The sta- tistical significance was defined as P-value $<0.05$.

Computer based statistical package for social sciences (SPSS) for windows 9.1 program was used.

\section{Results}

Table (1) shows that the mean $( \pm \mathrm{SD})$ age of the exposed group was $30.97 \pm 6.03$ years and that of the control group $31.68 \pm$ 7.882 , the mean $( \pm \mathrm{SD})$ duration of work of the exposed group was $6.25 \pm 4.433$ years.

The frequency distribution of the most relevant clinical manifestations detected through the detailed history (present and occupational) and clinical examination is shown in table (2).

Sensory disturbances were found in $26.7 \%$ of the exposed group, skin manifestations (dermatitis - redness and allergic dermatitis) in $30 \%$ and cardiovascular manifestations (arrhythmia mainly) in $50 \%$ (9.1\% of the control group). With regard to these clinical manifestations the differences between the exposed and the control groups were statistically significant.

Other manifestations (Headache, renal, respiratory and gastrointestinal) were higher in the exposed group compared to the controls but the difference was statistically insignificant. 
The mean $( \pm \mathrm{SD})$ of the kidney and liver function parameters are shown in table (3). No statistically significant difference was found between both groups. Estimation of serum MDA showed that the mean $( \pm \mathrm{SD})$ in the exposed group was $5.504 \mu$ $\mathrm{mol} / \mathrm{L} \pm 1.61$, versus $1.71 \mu \mathrm{mol} / \mathrm{L} \pm 0.68$ in the control group and the difference was statistically significant.

Cytogenetic chromosomal study was performed for all subjects who participated in the study. The means of the aberrations were higher in the exposed versus the con- trol subjects for all parameters, and the difference was statistically significant for gaps, isogaps, breaks and isobreaks.

Total aberrations in the exposed group were $13.4 \pm 4.3$ and in the control group were $4.4 \pm 2.1$ and the difference was statistically significant $(\mathrm{P}<0.05)$.

Table (5) shows correlations between the duration of work versus MDA level and versus chromosomal aberrations. Also correlation between serum MDA and chromosomal aberrations is shown. All were non significant correlations $(\mathrm{P}>0.05)$.

Table 1: Mean \pm SD of age and duration of work in the studied groups.

\begin{tabular}{|c|c|c|c|c|}
\hline & $\begin{array}{c}\text { Exposed } \\
\mathrm{n}=30\end{array}$ & $\begin{array}{c}\text { Control } \\
\mathrm{n}=22\end{array}$ & $\mathrm{t}$ & $\mathrm{P}$ \\
\hline Age & $30.97 \pm 6.03$ & $31.68 \pm 7.882$ & 0.371 & $>0.05$ \\
Duration of work & $6.25 \pm 4.433$ & & & \\
\hline
\end{tabular}


The mean $( \pm \mathrm{SD})$ of the kidney and liver function parameters are shown in table (3). No statistically significant difference was found between both groups. Estimation of serum MDA showed that the mean $( \pm \mathrm{SD})$ in the exposed group was $5.504 \mu$ $\mathrm{mol} / \mathrm{L} \pm 1.61$, versus $1.71 \mu \mathrm{mol} / \mathrm{L} \pm 0.68$ in the control group and the difference was statistically significant.

Cytogenetic chromosomal study was performed for all subjects who participated in the study. The means of the aberrations were higher in the exposed versus the con- trol subjects for all parameters, and the difference was statistically significant for gaps, isogaps, breaks and isobreaks.

Total aberrations in the exposed group were $13.4 \pm 4.3$ and in the control group were $4.4 \pm 2.1$ and the difference was statistically significant $(\mathrm{P}<0.05)$.

Table (5) shows correlations between the duration of work versus MDA level and versus chromosomal aberrations. Also correlation between serum MDA and chromosomal aberrations is shown. All were non significant correlations $(\mathrm{P}>0.05)$.

Table 1: Mean \pm SD of age and duration of work in the studied groups.

\begin{tabular}{|c|c|c|c|c|}
\hline & $\begin{array}{c}\text { Exposed } \\
\mathrm{n}=30\end{array}$ & $\begin{array}{c}\text { Control } \\
\mathrm{n}=22\end{array}$ & $\mathrm{t}$ & $\mathrm{P}$ \\
\hline Age & $30.97 \pm 6.03$ & $31.68 \pm 7.882$ & 0.371 & $>0.05$ \\
Duration of work & $6.25 \pm 4.433$ & & & \\
\hline
\end{tabular}


Table 2: Frequency distribution of clinical manifestation among exposed and control groups

\begin{tabular}{|l|c|c|c|c|c|c|}
\hline \multirow{2}{*}{} & \multicolumn{2}{|c|}{ Exposed } & \multicolumn{2}{c|}{ Control } & \multirow{2}{*}{$\mathrm{X}^{2}$} & \multirow{2}{*}{ P } \\
\cline { 2 - 5 } & No & $\%$ & No & $\%$ & & \\
\hline $\begin{array}{l}\text { 1- Neurological } \\
\text { a- Headache }\end{array}$ & 12 & 40.0 & 5 & 22.7 & 1.721 & $>0.05$ \\
\hline \multicolumn{1}{|c|}{ b- Sensory manifestation } & 8 & 26.7 & 0 & 0 & 6.933 & $<0.05^{*}$ \\
\hline 2- Cardiovascular & 15 & 50 & 2 & 9.1 & 9.65 & $<0.05^{*}$ \\
\hline 3- Respiratory manifestation & 8 & 26.7 & 3 & 13.6 & 1.292 & $>0.05$ \\
\hline 4- Renal & 1 & 3.3 & 0 & 0 & 0.748 & $>0.05$ \\
\hline 5- GIT & 9 & 30 & 2 & 9.1 & 3.32 & $>0.05$ \\
\hline 6- Skin & 9 & 30 & 0 & 0 & 7.981 & $<0.05^{*}$ \\
\hline 7-Musculoskeletal manifestation & 12 & 40.0 & 0 & 0 & 11.4 & $<0.05^{*}$ \\
\hline
\end{tabular}

Table 3: Mean \pm SD of liver and kidney function parameters, and serum malondialdehyde (MDA) in exposed and control groups.

\begin{tabular}{|l|c|c|c|c|}
\hline & Exposed & Control & $\mathrm{t}$ & $\mathrm{P}$ \\
\hline MDA $(\mu \mathrm{mol} / \mathrm{L})$ & $5.504 \pm 1.61$ & $1.71 \pm 0.68$ & 10.32 & $<0.05^{*}$ \\
\hline Total bilirubin $(\mathrm{mg} / \mathrm{dl})$ & $0.314 \pm 0.183$ & $0.424 \pm 0.296$ & 1.659 & $>0.05$ \\
\hline ALT $($ Unit/L) & $17.93 \pm 5.64$ & $18.23 \pm 4.59$ & 0.203 & $>0.05$ \\
\hline AST $($ Unit/L) & $28.2 \pm 9.1$ & $25.62 \pm 5.1$ & 1.215 & $>0.05$ \\
\hline Alkaline phosphatase $($ Unit/L) & $119.83 \pm 28.8$ & $121.09 \pm 33.35$ & 0.145 & $>0.05$ \\
\hline Urea $(\mathrm{mg} / \mathrm{dl})$ & $25.1 \pm 7.043$ & $23.64 \pm 6.91$ & 0.746 & $>0.05$ \\
\hline Creatinine $(\mathrm{mg} / \mathrm{dl})$ & $0.69 \pm 0.16$ & $0.567 \pm 0.26$ & 2.17 & $>0.05$ \\
\hline
\end{tabular}


Table 4: Mean \pm SD of chromosomal aberrations in both exposed and control groups:

\begin{tabular}{|l|c|c|c|c|}
\hline & Exposed & Control & $\mathrm{t}$ & $\mathrm{P}$ \\
\hline Gaps & $8.07 \pm 2.3$ & $2.00 \pm 1.049$ & 11.06 & $<0.05$ \\
\hline Iso gaps & $2.79 \pm 1.67$ & $1.09 \pm 0.831$ & 3.202 & $<0.05$ \\
\hline Breaks & $4.73 \pm 1.76$ & $1.15 \pm 0.376$ & 7.214 & $<0.05$ \\
\hline Iso breaks & $1.76 \pm 0.76$ & $0.50 \pm 0.57$ & 3.1 & $<0.05$ \\
\hline Fragments & $1.5 \pm 0.67$ & $0.50 \pm 0.707$ & 1.93 & $>0.05$ \\
\hline Deletion & $1.47 \pm 0.874$ & $0.8 \pm 0.447$ & 1.63 & $>0.05$ \\
\hline Separation & $1.00 \pm 0.0$ & $0.6 \pm 0.548$ & 1.63 & $>0.05$ \\
\hline Total & $13.4 \pm 4.3$ & $4.4 \pm 2.1$ & 28.5 & $<0.05$ \\
\hline
\end{tabular}

Table 5: Correlation between duration of work on one hand and MDA and chromosomal aberrations on the other and between MDA and chromosomal aberrations

\begin{tabular}{|l|c|c|}
\hline & $\mathrm{r}$ & $\mathrm{p}$ \\
\hline Duration of work + malondialdehyde & 0.45 & $>0.05$ \\
\hline Duration of work + chromosomal aberrations & 0.170 & $>0.05$ \\
\hline Malondialdehyde + chromosomal aberrations & 0.227 & $>0.05$ \\
\hline
\end{tabular}




\section{Discussion}

Most of the data regarding health effects of chloroform in humans were obtained from clinical reports describing health effects in patients under chloroform anesthesia or from experimental studies on animals.

Clinical reports indicate that the central nervous system, cardiovascular system, liver and kidneys are the target organs of chloroform toxicity after inhalation or oral exposure (Maffei et al., 2005).

Respiratory and gastrointestinal effects have also been reported (Harris and Groh, 1985). Studies, in animals, also indicated that chloroform exposure may induce reproductive and developmental effects and cause cancer (Dodds and King, 2001).

Chloroform toxicity can be attributed to the presence of the parent compound and the formation of phosgene metabolite, which reacts with molecules such as cysteine to deplete hepatic glutathione (Pohl et al., 1981) or form adducts with microsomal proteins (Corley et al., 1990).

There are several possible mechanisms for chloroform toxicity, for example, neurotoxic and respiratory effects of chloroform may be attributed to the interaction with gangliosides in neuronal membranes (Harris and Groh, 1985) and phospholipids in the surfactant monolayer of the lower respiratory tract (Enhorning et al., 1986).

Another proposed reaction of chloroform and lipids would result in the formation of conjugated diens which are indicative of lipid peroxidation (De Groot and Noll, 1989).

This study aimed at investigating hepatorenal, and genetic health effects of chloroform, and also studying the possible role of lipid peroxidation as one of the mechanisms of action of chloroform and its role in inducing its adverse health effects.

The studied group was composed of 32 female, non-smokers, with a mean age of $30.97+6.03$ years, and mean duration of work of $6.25+4.433$ years. They were occupationally exposed to chloroform as the main laboratory chemical used with the HPLC, in Analgesic and Narcotic Drug analysis laboratory in the [NODCAR]. An age, sex, and socially matched group of 22 individuals were taken as controls.

A comprehensive medical questionnaire, clinical examinations, biochemical investigations to assess hepatorenal functions and serum MDA (a lipid peroxidation predict) were done to each individual. In addition, cytogenetic study was carried out to estimate the chromosomal aberrations. 
Dermal effects of chloroform were reported as early as 1968 when Malten et al., observed the complete destruction of the stratum corneum in the skin of 2 young volunteers exposed to chloroform.

Similar results were observed by King (1993) in his clinical study on 21 females and 21 males exposed to chloroform topically applied, the exposed subjects experienced a burning sensation in the skin.

In the current study skin manifestation (redness, drying, cracking, itching and allergic dermatitis) were reported in $30 \%$ of the exposed subjects which was statistically significant when compared with the control group.

According to Genium (1992), chronic occupational dermal exposure to chloroform may cause redness, dryness and cracking of the skin.

Similar conclusion was reached by $\mathrm{Xu}$ and Weisel (2005).

Headache, and sensory manifestations were detected in $40 \%$ and $26.7 \%$ of the exposed group, respectively, versus $22.7 \%$ and $0 \%$ of the control group, with statistically significant difference for the sensory manifestations $(\mathrm{P}<0.05)$. These results are in agreement with Meldrum (1991) who, at chronic low-level occupational exposure to chloroform, reported a variety of minor complaints including headache, lassitude, depression and digestive disturbances.

In humans, neurological effects of inhalation exposure to chloroform are well documented because chloroform has been used as an anesthetic for surgery. Chloroform at high concentrations acts as a depressant of the CNS (Erdinger et al., 2004). Chronic low-level occupational exposure to chloroform resulted in exhaustion, lack of concentration depression and irritability (Sitting, 1991).

Similar results were found by $\mathrm{Li}$ et al. (1993) who studied the health effects of chloroform on 61 workers exposed for 115 years to concentrations of chloroform ranging from 0.87 to $28.9 \mathrm{ppm}$.

Chloroform intoxication can lead to death caused by depression of the central nervous system, fatal arrhythmias, or respiratory arrest.

Several cases of fatal arrhythmia after intoxication with chloroform have been described, and the electrophysiological basis of the arrythmogenic potential of chloroform has been related to inactivation of the HERG potassium channels (Scholz et.al., 2006).

In this study, $50 \%$ of the studied exposed subjects had cardio-vascular manifestations (mainly arrhythmia as bradycar- 
dia, syncopal attacks, extrasystoles) compared to $9 \%$ of the control subjects, the difference is statistically significant.

Chloroform directly and dosedependently provokes muscle contraction in the respiratory tract in experimental animals, which may result from the activation of ryano dine receptor $\mathrm{Ca} 2+$ release channel in sacroplasmic reticulum and may not depend on the muscarinic and adrenergic activation of $\mathrm{Ca} 2+$ entry from the extracellular environment (Lin et al., 2002).

Respiratory complaints (Cough, phlegm, asthma) were found in $26.7 \%$ of the exposed group versus $9.1 \%$ of the controls, the difference was statistically insignificant.

Larson et al. (1996) investigated in their experimental study of female mice, the toxicity of chloroform vapors at various concentrations ranging from 0 to 90 ppm. Although no clinical respiratory manifestations were detected, pathological changes (proliferative responses in the periostium of the nasal bones) were found in the sacrificed animals after exposure to $\geq$ $10 \mathrm{ppm}$.

In this study GIT symptoms (nausea dyspepsia - flatulence) were detected in $30 \%$ of the workers versus $9.1 \%$ in the controls but the difference was statistically insignificant. This was not in agreement with Phoon et al. (1983) who observed that nausea and vomiting were significantly observed in workers exposed to chloroform (14 - $400 \mathrm{ppm}$ for 1-6 months) and they related these manifestations to toxic hepatitis.

Table (3) shows the results of some liver and kidney function parameters in both the exposed and control groups. All parameters showed no statistically significant differences between exposed and control groups. Chloroform-induced hepatic and renal toxicity are among the major toxic effects observed in both humans and animals after exposure to chloroform.

In contrast to our results Phoon et al. (1983) found abnormal liver function parameters in workers exposed to $14-400$ ppm of chloroform for periods ranging from 1-6 months. They also found significant clinical manifestations suggestive of toxic hepatitis.

However, in agreement with our results are those of Aiking et al. (1994), who examined the possible hepatotoxicity of chloroform exposure in competitive swimmers who trained in indoor chlorinated swimming pools compared to a control group, no significant difference was observed in liver functions. 
Also Li et al. (1993) found no biochemical renal abnormalities in factory workers in china exposed to varying levels of chloroform.

However, despite no statistically significant difference in liver enzymes, the study of Aiking et al.,(1994) did determine that B2-microglobulin was elevated in the indoor pool swimmers, suggesting some degree of renal damage due to higher inhaled air concentration to chloroform present in the air of indoor swimming pools.

The ability of chloroform to produce toxicity and regenerative cell proliferation in the kidney of mice was examined by Larson et al. (1996). Lesions were found microscopically mainly in the epithelial cells of the proximal convoluted tubules, mineralization of the cortex and enlarged nuclei in the epithelial cells were also noted.

The mechanism of chloroform and carbon tetrachloride toxicity on mouse hepatocytes were investigated. The cytotoxicity of both compounds was dose and duration dependent. The researchers found that the metabolism of these compounds and production of free radicals play the major role in their toxicity (McCulloch, 2003).
Lipid peroxidation is a well established mechanism of cellular injury in plants and animals, and is used as an indicator of oxidative stress in cells and tissues. Lipid peroxides are unstable and decompose to form a complex series of compounds. Polyunsaturated fatty acid peroxides generate malondialdehyde (MDA) and 4-hydroxyalkenals (HAE) upon decomposition. Therefore measurement of MDA and HAE has been used as indicators of lipid peroxidation (Draper et al., 2000 and Sorensen et al., 2003).

In this study, the level of MDA in serum of both exposed and control was 5.504 $\mu \mathrm{mol} / \mathrm{L} \pm 1.61$ and $1.71 \mu \mathrm{mol} / \mathrm{L} \pm 0.68$, respectively and the difference was statistically significant $(\mathrm{P}<0.05)$.

These results are in agreement with Ekström et al. (1988) who used malondialdehyde excretion in urine as an index of the toxicological effects of chloroform. The researchers concluded that MDA assay was a selective and accurate marker for the toxicity induced by chloroform in exposed workers.

Also in agreement with our results are the results of Beddowes et al. (2003) in their study to investigate the genotoxic potential and the mechanism of action of chloroform and carbon tetrachloride. They deduced that chloroform can induce an ox- 
idative stress via depletion of glutathione (GSH) and other antioxidant defenses and that this may lead to indirect genotoxicity. They found significant increase of DNA strand breakage, lipid peroxidation and GSH depletion.

Similar results were concluded by Yuan et al. (2005) in their experimental study.

Table (4) shows means + SD of chromosomal aberrations in both exposed and control groups. All aberrations were higher in the exposed workers and the difference was statistically significant in most aberrations.

Although there were clearly positive results in a few genotoxic assays, results for chloroform were more frequently found to be negative than positive in genotoxic tests and it is even considered by some researchers as a non-genotoxic or epigenetic carcinogen.

According to Yuan et al. (2005) the genotoxic effect of chloroform is mainly through metabolism of chloroform in the liver to the highly reactive phosgene, a plausible alkylating agent that binds to cellular proteins, lipid, and DNA and is attributed to a lesser extent to a direct, weak genotoxic activity of chloroform.
Our results are in agreement with Araki et al. (2004) who studied the in vitro mutagenic and clastogenic activity of chloroform and carbon tetrachloride.

Also in agreement with our results are those of Brennan and Schiestle (1998), who found, in addition, that the genotoxic potential is induced via oxidative free radical species as proven by the fact that the free radical scavenger $\mathrm{N}$-acetyl cysteine reduced chloroform-induced genotoxicity and chromosomal recombination.

Lu et al. (1999) mentioned that exposure to chloroform in experimental animals resulted in an increase in DNA breakage and he found that MDA levels were significantly increased in all studied animals and in all organs tested.

However, negative results for genotoxicity were reported by Lévesque et al. (2002) who studied the effect of chloroform used in the house hold. The difference may be attributed to differences in the purity of chloroform to which the studied groups were exposed, duration and frequency of exposure.

When correlating duration of work (exposure) with MDA level and chromosomal aberrations, the correlation was insignificant. Also correlation of MDA and total chromosomal aberrations was insignificant. 
These results, although not expected, can be explained by the fact that there are many proposed mechanism for chloroform toxicity as stated by McCulloch in 2003:

1- Lack of direct in vivo or in vitro genotoxicity.

2- Cytotoxicity and compensatory cell proliferation in the affected organs.

3- Cytolethality leads to chronically stimulated cell proliferation and related events such as inflammation and growth stimulation. Consequently lipid peroxidation and glutathione depletion are not the only mechanisms for genotoxcity.

Also exposure to chemical agents and their toxic effects are dependent upon many factors as the time spent in the working environment, the concentration of the chemical and personal activities as well as personal general health, liver status and the use of personal protection (Faiola et al., 2004).

\section{References}

1. ACGIH (1994): Threshold limit values for chemical substances and physical agents and biological exposure indices. American Conference of Governmental Industrial Hygienist, $\mathrm{OH}$ Cincinnati.

2. Aiking, H., Van Acker, M.B., Scholten, R.J., Feenstra, J.F. and Valkenburg, H.A. (1994): Swimming pool chlorination: a health hazard? J. Toxiol. Lett., 72 (1-3): 375-380.
3. Araki, A., Kamigaito, N., Sasaki,T. and Matsushima, T. (2004): Mutagencity of carbon tetrachloride and chloroform in salmonella typhimurium TA98, TA 100, TA 1535 and TA1537, and Escherichia coli WP2uvrA/pKM101 and WP2/ pKM101,using a gas exposure method. Environ. Mol. Mutagen., 43 (2): 128-133.

4. Beddowes, E.J., Faux, S.P. and Chipman, J.K. (2003): Chloroform, carbon tetrachloride and glutathione depletion induce secondary genotoxicity in liver cells via oxidative stress. Toxicology, 187 (2-3): 101-115.

5. Brennan, R.J. and Schiestl, R.H. (1998): Chloroform and carbon tetrachloride induce intrachromosomal recombination and oxidative free radicals in Saccharomyces cerevisiae. Mutat. Res., 397 (2): 271-278.

6. Corley, R.A., Mendrala, A.L.,Smith, F.A. , Staats, D.A., Gargas, M.L., Conolly, R.B., Andersen, M.E. and Reitz, R.H. (1990): Development of a physiologically based pharmacokinetic model for chloroform. Toxicol. Appl. Pharmacol., 103 (3):512-527.

7. De Groot, H. and Noll, T. (1989): Halomethane hepatotoxicity: Induction of lipid peroxidation and inactivation of cytochrome $\mathrm{P} 450$ in rat liver microsomes under low oxygen partial pressures. Toxicol. Appl. Pharmacol., 97 (3): 530-537.

8. Dodds, L. and King, W.D. (2001): Relation between trihalomethane compounds and birth defects. Occup. Environ. Med., 58: 443.

9. Draper, H.H., Csallany, A.S. and Hadley, M. (2000): Urinary aldehydes as indicators of lipid peroxidation in vivo. Free Radic. Boil. Med., 29 (11): 1071-7. 
10. Ekström, T., Warholm, M., Kronevi, T. and Högberg, J. (1988): Recovery of MDA in urine as a 2,4 dinitrophenylhydrazine derivative after exposure to chloroform or hydroquinone. Chem. Boil. Interact., 67 (1-2): 25-31.

11. Enhorning, G., Pototschnik, R., Possmayer F. and Burgoyne, R. (1986): Pulmonary surfactant films affected by solvent vapors. Anesth. Analg., 65: 1275-1280.

12. Erdinger, L., Kuhn, K.P., Kirsch, F., Feldhues, R., Frobel, T, Nohynek, B. and Gabrio, T. (2004): Pathways of trihalomethane uptake in swimming pools. Int. J. Hyg. Environ. Health, 207 (6): 571-575.

13. Faiola, B., Fuller, E.S., Wong, V.A. and Recio, L. (2004): Gene expression profile in bone marrow and hemopoietic stem cells in mice exposed to inhaled benzene . Mut . Res. 549 :195-212.

14. Gemma, S., Vittozzi, L. and Testai, E. (2003): Metabolism of Chloroform in the human liver and identification of the competent P450s. Drug Metab., 31 (3): 266-74.

15. Genium, C. (1992): Material safety data sheet No 315. Scectady Ny: Genium publishing corporation.

16. Harris, R.A. and Groh, G.I. (1985): Membrane disordering effects of anesthetics are enhanced by gangliosides. Anesthesiol., 62 (2): 115 - 119.

17. King, R.B. (1993): Topical aspirin in chloroform and the relief of pain due to herpes zoster and postherpetic neuralgia. Arch. Neurol., 50: 10461053.

18. Larson, J.L., Templin, M.V., Wolf, D.C., Jamison, K.C., Leininger, J.R., Mery, S., Morgan, K.T., Wong, B.A., Conolly, R.B., and Butter- worth, B.E. (1996): A 90- day chloroform inhalation study in female and male B6 C3F1 mice: implications for cancer risk assessment. Fundam. Appl. Toxicol., 30 (1): 118-137.

19. Lévesque, B., Ayotte, P., Tardif, R., Ferron, L., Gingras, S., Schlouch, E., Gingras, G., Levallois, P. and Dewailly, E. (2002): Cancer risk associated with household exposure to chloroform. J. Toxicol. Environ. Health A., 65(7):489-502.

20. Li, L.H., Jiang, X.Z., Liang, Y.X., Chen, Z.Q., Zhou, Y.F. and Wang, Y.L. (1993): Studies of the toxicity and maximum allowable concentration of chloroform. Biomed. Environ. Sci., 6 (2): 179-186.

21. Lin, Y.R., Chen, H.H. and Chan, M.H. (2002): Role of ryanodine receptors in chloroform - induced contraction in swine tracheal smooth muscle. Toxicol. Appl. Pharmacol., 183 (2): 146151.

22. Lu, W., Yue, F., Chen, X. and Li, X. (1999): Genotoxicity and lipid peroxidation caused by organic extracts of chlorinated drinking water in rats and HepG2 cells. Nov; 28(6):326-8.

23. Maffei, F., Buschini, A., Rossi, C., Forti, G.C. and Hrelia, P. (2005): Use of comet test and micronucleus assay on human white blood cells for in vitro assessment of genotoxicity induced by different drinking water disinfection protocols. Environ. Mol. Mutagen., 46 (2): 116-125.

24. Malten, K.E., Spruit, D., Boemaars, H.G. and de Keizer, MJ (1968): Horny layer injury by solvents. Berufsdermatosen, 16 (3): 135-147.

25. McCulloch, A. (2003): Chloroform in the environment: occurrence, sources, sinks and effects. Chemosphere, 50 (10): 1291-1308. 
26. Meek, M.E., Beauchamp, R., Long, G., Moir, D., Turner, L. and Walker, M. (2002): Chloroform: Exposure estimation, hazard characterization, and exposure response analysis. J. Toxicol. Environ. Health B Crit. Rev., 5 (3): 283-334.

27. Meldrum, M. (1991): Chloroform. HSE Toxicity Review, 23: P.36

28. Phoon, W.H., Goh, K.T., Lee, L.T., Tan, K.T. and Kwok, S.F. (1983): Toxic Jaundice from occupational exposure to chloroform. Med. J.M., 38 (1): $31-34$.

29. Pohl, L.R., Branchfloser, R.V. , Highet, R.J., Martin, J.L., Nunn, D.S., Monks, T.J., George, J.W., and Hinson, J.A. (1981): The formation of diglutathionyl dithiocarbonate as a metabolite of chloroform, bromotrichloromethane, and carbon tetrachloride. Drug Metab. Dispos., 9: 334-339.

30. Rosenberg, J. and Katz, E. (2004): Solvents. Current Occupational and Environmental Medicine, 4th edition, Joseph LaDou ed. A Lang Medical book- McGrow Hill Companies.

31. Scholz, E.P., Alter, M., Zitron, E., Kiesecker, C., Kathofer, S., Thomas, D., Kreye, V.A., Kreuzer, J., Becker, R., Katus, H.A., Greten, J. and Karle, C.A. (2006): In vitro modulation of HERG channels by organochlorine solvent trichlormethane as potential explanation for proarrhythmic effects of chloroform. Toxicol. Lett. 165(2):156166.

32. Sitting, M. (1991): Handbook of toxic and hazardous chemicals. 3rd Edition, Park Ridge Ed. Noyes publications.

33. Sorensen, M., Skov, H., Autrup, H., Hertel, O and Loft, S. (2003): Urban benzene exposure and oxidative DNA damage : Influence of genetic polymorphisms in metabolism genes. Sci. Total Environ., 309 (1-3): 69-80.

34. Stringer, M.D., Corog, P.G. and Freeman, A. (1989): Lipid peroxidation and atherosclerosis. B.M.J., 281-298.

35. Verma, J. and Babu, L. (1989): Human chromosome. Manual of basic technique. 1st ed. Permagon press.

36. Xu, X. and Weisel, C.P. (2005): Dermal uptake of chloroform and haloketones during bathing $\mathrm{J}$. Expo. Anal. Environ. Epidemiol., 15 (4): 289296.

37. Yuan, J., Wu, X.J., Lu, W.Q., Cheng, X.L., Chen, D., Li, X.Y., Liu, A.L., Wu, J.J., Xie, H., Stahl, T. and Mersch-Sundermann, V. (2005): Chlorinated river and lake water extract caused oxidative damage, DNA migration and cytotoxicity in human cells. Int. J. Hyg. Environ. Health, 208(6): 481 - 488. 